\title{
Design of a Cooper pair box electrometer for application to solid-state and astroparticle physics
}

\author{
L. Tosi, ${ }^{1}$ D. Vion, ${ }^{2}$ and H. le Sueur ${ }^{1, *}$ \\ ${ }^{1}$ CSNSM, Univ. Paris-Sud, CNRS/IN2P3, Université Paris-Saclay, 91405 Orsay, France \\ ${ }^{2}$ SPEC, CEA, CNRS, Université Paris-Saclay, CEA Saclay, 91191 Gif-sur-Yvette, France
}

\begin{abstract}
We describe the design and principle of operation of a fast and sensitive electrometer operated at millikelvin temperatures, which aims at replacing conventional semiconducting charge amplifiers in experiments needing low back-action or high sensitivity. The electrometer consists of a Cooper Pair box (CPB) coupled to a microwave resonator, which converts charge variations to resonance frequency shifts. We analyze the dependence of the sensitivity on the various parameters of the device, and derive their optimization. By exploiting the nonlinearities of this electrometer, and using conventional nanofabrication and measurement techniques, a charge sensitivity of a few $10^{-7} e / \sqrt{\mathrm{Hz}}$ can be achieved which outperforms existing single charge electrometers.
\end{abstract}

\section{INTRODUCTION}

The detection of the time variations of minute amounts of charge is instrumental in the success of recent experiments in many fields. We can cite, for instance, the measurement of the transport statistics in a mesoscopic circuit [1, the demonstration of exotic states of matter such as Majorana fermions 2, or even the observation of new phenomena in astrophysics such as neutrino coherent scattering [3, 4] or the search for direct dark matter detection at low masses [5].

Such ultimate charge sensing requires to operate at low enough temperatures in order to suppress the thermal charge fluctuations that would mask the desired phenomena. These experiments are typically performed in dilution refrigerators at temperatures around 10-50 $\mathrm{mK}$. In this temperature range, conventional semiconducting charge amplifiers generally do no work or they dissipate more than the refrigerator can handle. Secluding the readout electronics at higher temperature is neither desired because it unavoidably adds stray capacitances that reduce the charge-detection bandwith and increase the noise pick-up.

It has long been shown that single charge mesoscopic devices (SCD) are well suited for ultimate electrometry at these temperatures. Some of these devices are actually the dual of the SQUID and as such, they are also potentially quantum limited. The most famous SCDs are the Single Electron Transistor or quantum dots that are operated essentially as field transistors: the gate charge modulates the out-of-equilibrium (dissipative) current flowing between source and drain. Such out-of equilibrium operation creates many electron-hole pairs nearby the SCD, that will eventually relax by emitting phonons or photons into the environment. These radiations in turn influence the charge to be detected in a sensitive device. It is often difficult to assess the influence that those microscopic excitations can have onto the measured systems. Fortunately, such uncontrolled electromagnetic back-action can be completely avoided by using superconducting ver- sions of SCDs, such as the single Cooper pair transistor $(\mathrm{CPT})$ or its simpler version the Cooper pair box (CPB). Indeed, these devices can be operated in equilibrium (no voltage bias and no dissipation), making their back-action minimal and through a well-identified channel.

Finally, large-bandwidth implementations [6, 7] of these mesoscopic charge sensing devices were demonstrated by coupling them with resonant microwave $(\mu \mathrm{w})$ circuits. This further enables the frequency-multiplexing of charge detectors on a single line which is highly desirable in experiments where many charge channels need to be monitored. Hence our choice to use an optimized microwave superconducting, i.e. non dissipative, electrometer, which has both large bandwidths and multiplexing capabilities, and with a minimal back-action.

In this work we analyse in depth the optimal design of a $\mu \mathrm{w}$ Cooper pair box electrometer suitable for a large variety of applications, including the charge channel of bolometric particle detectors. Our optimization takes advantage of the quantum nature of the coupled resonator - CPB system and it includes the analysis of nonlinearities, that are shown to enhance the performances by one order of magnitude compared to state-of-the-art [8] 10 .

In section 2 we describe our electrometer based on a $\mathrm{CPB}$ embedded in a microwave resonator. The electrometer's small signals sensitivity is derived in section 3 , first in the linear regime of the $\mathrm{CPB}$, then taking into account the $\mathrm{CPB}$ nonlinear response, and finally including the resonator quantum nonlinearities. Section 4 addresses the main sources of imperfections, i.e. the problem of offset charge noise and quasiparticle poisonning of the island [11. Section 5 is a brief discussion about how to integrate our electrometer in a detector. 

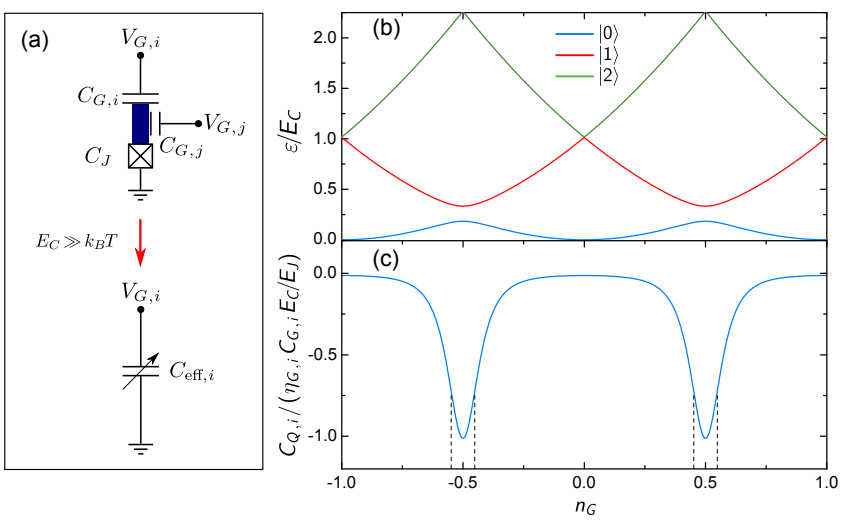

Figure 1. The CPB and its effective capacitance. (a) Circuit representation of the CPB: an island (dark rectangle) connected to the ground through a Josephson junction, and capacitively coupled to one or several "gate" electrodes. As seen from a particular gate $i$ the circuit is equivalent to an effective variable capacitance $C_{\text {eff }, i}$ when $E_{C}$ is large compared to $k_{B} T$. (b) CPB's first 3 energy levels computed numerically for $E_{J} / E_{C}=0.2$ as a function of the reduced charge $n_{G}$ accumulated on the gates. (c) Corresponding quantum capacitance $C_{Q}$ of the ground state of the CPB (relative to the absolute maximum at $n_{G}=1 / 2$ ). Vertical dashed lines indicate the working gate charge $n_{G}^{\text {opt }}$ at which sensitivity to a small change in $n_{G}$ is optimum.

\section{THE RF-CPB ELECTROMETER}

\section{A. The Cooper pair box}

The Cooper pair box (CPB - see fig. 1 a) 12 15, consists of a micron-sized superconducting "island" connected to a much larger superconducting "reservoir" through a Josephson junction. The island can be capacitively coupled to other electrodes to sense the charge that they carry.

The CPB is characterized by a single degree of freedom: the charge on its island and its conjugate variable the phase across the junction. Its behavior results from a competition between charging effects which tend to localize charge in the island, and the Josephson effect that allows Cooper pairs to tunnel in and out. Both are quantified by their characteristic energy: (i) $E_{C}=(2 e)^{2} / 2 C_{\Sigma}$ the charging energy of one Cooper pair, with $C_{\Sigma}=C_{J}+\sum_{i} C_{G, i}$ the sum of the Josephson junction capacitance $C_{J}$ and all the capacitances $C_{G, i}$ between the island and gates $i$; (ii) $E_{J}=\Phi_{0} I_{C} / 2 \pi$ the Josephson energy coupling two charge states of the island differing by one Cooper pair, with $\Phi_{0}=h / 2 e$ the magnetic flux quantum and $I_{C}$ the critical current of the junction.

\section{The CPB Hamiltonian}

The CPB Hamiltonian, written in the basis of charge states $|n\rangle$ corresponding to integer numbers $n$ of Cooper pairs on the island, reads

$$
\begin{aligned}
H_{\mathrm{CPB}}=\sum_{n=-\infty}^{+\infty}\left\{E_{C}\left(\hat{n}-n_{G}\right)^{2}|n\rangle\langle n|\right. \\
\left.\quad-\frac{E_{J}}{2}(|n+1\rangle\langle n|+| n\rangle\langle n+1|)\right\},
\end{aligned}
$$

where the "gate charge" $n_{G}=\sum n_{G, i}+n_{G}$ is the sum of all reduced charges $n_{G, i}=C_{G, i} V_{G, i} / 2 e$ of the surrounding gate electrodes at voltages $V_{G, i}$, plus a fluctuating offset charge $n_{G}$ of microscopic origin. $H_{\mathrm{CPB}}$ can be diagonalized either numerically by truncating the charge basis, or analytically in the conjugate phase basis [16]. The eigenstates of the CPB are coherent superpositions of charge states forming 2e-periodic energy bands $\varepsilon_{k}\left(n_{G}\right)$, the shape of which depends on the $E_{J} / E_{C}$ ratio. In the so-called charge regime $E_{J} \ll E_{C}$ of interest for electrometry, the CPB states are close to pure charge states and their energy and other observables depend strongly on $n_{G}$ (see Fig. 1 b).

2. Our observable: the quantum capacitance in the charge regime

As can be seen from the CPB spectrum on Fig. 1.b, the degeneracy between two neighbouring charge states is lifted around $n_{G}=1 / 2$ by the Josephson coupling. This induces a change of curvature $\partial_{n_{G}}^{2} \varepsilon_{0}$ of the CPB ground state. This curvature, which is plotted in Fig. 1.c, enters the capacitance to ground of any particular gate electrode $i$, by a quantity $C_{Q, i} \equiv-\eta_{i} C_{G, i} \partial_{n_{G}}^{2} \varepsilon_{0}$ called the quantum or Josephson capacitance of the CPB [1719, with $\eta_{i}=C_{G, i} / C_{\Sigma}$ the gate $i$ lever arm.

The quantum capacitance is obviously higher in the charge regime: For $E_{J} / E_{C} \lesssim 0.1$, a subspace of two charge states accurately describes the system, and

$$
C_{Q, i} \simeq-\eta_{i} C_{G, i} \frac{E_{C} E_{J}^{2}}{\left[E_{C}^{2}\left(1-2 n_{G}\right)^{2}+E_{J}^{2}\right]^{3 / 2}}
$$

can even exceed $C_{\Sigma}$. It can be used for instance to probe the state of the CPB charge qubit [20].

The modulation of $C_{Q}$ with gate charge $n_{G}$ also enables charge sensing: charge fluctuations on a gate can indeed be detected by monitoring the CPB's effective capacitance, provided that thermal excitations of the CPB at all $n_{G}$ are avoided by operating it at $k_{B} T \ll \min \left(\varepsilon_{1}-\varepsilon_{0}\right)=$ $E_{J}$. Moreover, because $C_{Q, i}$ can be measured in ac at relatively high frequency, it is a good observable for highspeed electrometry. 


\section{B. Measurement scheme: Embedding the CPB in a resonator}

To measure its quantum capacitance variations, the CPB is capacitively coupled to a superconducting LC resonator of frequency $\nu_{r}=1 / 2 \pi \sqrt{L_{r} C_{r}}$ and impedance $Z_{r}=\sqrt{L_{r} / C_{r}}$ with a coupling capacitance $C_{G, r}$ (see Fig. 2]. According to previous section, the resonator is thus terminated by an effective tunable capacitance $C_{\text {eff }}=C_{\text {geom }, r}+C_{Q, r}\left(n_{G}\right)$ (see Fig. 1). Here, $C_{\text {geom }, r}=$ $\left(1-\eta_{G, r}\right) C_{G, r}$ is the geometrical capacitance seen from the microwave port of the CPB. Classically, $C_{\text {eff }}$ adds to the resonator capacitance $C_{r}$ and displaces its bare frequency $\nu_{r}$ to $\tilde{\nu}_{r}\left(n_{G}\right)=\nu_{r}\left(1+C_{\text {eff }}\left(n_{G}\right) / C_{r}\right)^{-1 / 2}$.

The resonator is coupled inductively to the side of an on-chip transmission line of characteristic impedance $Z_{0}=50 \Omega$ whose complex transmission $S_{21}(\nu=\omega / 2 \pi)=$ $V_{2}^{-} / V_{1}^{+}$at frequency $\nu$ is measured close to the resonator frequency $\tilde{\nu}_{r}$ (see Fig. 2). The frequency shift of the resonator induced by the $\mathrm{CPB}$ modifies the phase and amplitude of $S_{21}$. The output signal at port 2 is then amplified by a first amplifier stage whose input noise determines the overall sensitivity of the setup, as discussed below.

This "side-coupling" is inspired from detector arrays for astroparticle and astronomy, and is used in a variety of applications including arrays of RF-SETs [21, kinetic inductance detectors (KIDs) 22], SQUIDs [23] and qubits 24. It can provide up to a few $\mathrm{MHz}$ bandwidth and enables multiplexing a large number of detectors on a single microwave line.

\section{SMALL SIGNAL SENSITIVITY}

We now derive the expressions for the sensitivity $S_{e}$ of our electrometer. In sections III A to IIID we first make a simple calculation that neglects the nonlinearity of the resonator inherited from its coupling to the CPB. In section III E we take this effect into account, and show how the nonlinearity can be exploited to improve the sensitivity. We finally define the procedure to find the optimal parameters.

The electrometer responsivity, defined as the modulus of the $S_{21}$ derivative with respect to $n_{G}$,

$$
\begin{aligned}
\left|\partial_{n_{G}} S_{21}\right|= & \left|\partial_{\nu} S_{21}\right| \cdot \partial_{C_{Q}} \nu_{r} \cdot \partial_{n_{G}} C_{Q, r} \\
& {[V / 2 e / \text { input } V] }
\end{aligned}
$$

is simply the product of the variation of $S_{21}$ with frequency, the variation $\partial_{C} \nu_{r}=\partial_{C_{Q}} \nu_{r}$ of the resonance frequency with capacitance, and the variation of $C_{Q}$ with gate charge. This expression is valid in the linear regime, when the $\mu \mathrm{w}$ probe signal represents small variations $\delta n_{G} \ll 1$ of $n_{G}$. (a)

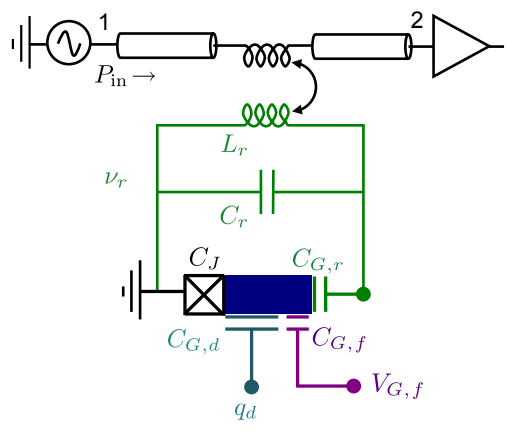

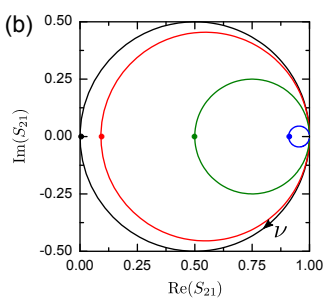

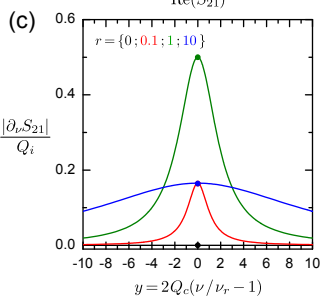

Figure 2. Microwave CPB electrometer. (a) The resonator embedding the CPB is "side-coupled" inductively to a $50 \Omega$ transmission line along which the $\mu \mathrm{w}$ excitation propagates and is amplified. The CPB quantum capacitance $C_{Q}$ seen from the $\mu \mathrm{w}$ gate acts as a parallel capacitance that adds up to the bare resonator capacitance $C_{r}$. The charge $q_{d}$ collected on the detector gate capacitor $C_{G, d}$ modifies $C_{Q}\left(n_{G}\right)$ and shifts the resonator frequency $\nu_{r}$. To maximize the response to charge variations a "feedback" gate (labelled $f$ ) is used to maintain $n_{G}$ at the optimal value for which $\partial_{n_{G}} C_{Q}$ is maximum. (b) The resonator is probed through its $S_{21}$ complex transmission coefficient between ports 1 and 2 . When varying the frequency $S_{21}$ moves in the complex plane along a circle starting from and ending at $\operatorname{Re}\left(S_{21}\right)=1$ far away from resonance, and passing through a minimum amplitude at resonance (dots); the diameter of this circle depends on the ratio $Q_{c} / Q_{i}$ of the internal losses to the energy leakage towards the line. (c) Linear responsivity $\left|\partial_{\nu} S_{21}\right|$ of the resonator (see section IIIB plotted as a function of reduced frequency $y$, for the same set of $r=Q_{c} / Q_{i}$. Responsivity is maximal at resonance $(y=0)$ and for critical coupling $(r=1)$, and increases with $Q_{i}$.

\section{A. Capacitance response $\partial_{n_{G}} C_{Q, \mathrm{rf}}$}

The capacitance derivative with respect to charge can be expressed analytically from Eq. (2) for $E_{J} / E_{C} \lesssim 0.1$ and small $\mu \mathrm{w}$ power:

$$
\partial_{n_{G}} C_{Q, r} \simeq \eta_{G, r} C_{G, r} \frac{6 E_{C}^{3} E_{J}^{2}\left(2 n_{G}-1\right)}{\left[E_{C}^{2}\left(1-2 n_{G}\right)^{2}+E_{J}^{2}\right]^{5 / 2}}
$$

It takes a maximum $\propto\left(E_{C} / E_{J}\right)^{2}$ at $n_{G}^{\text {opt }}=1 / 2 \pm$ $E_{J} / 4 E_{C}$. This value of $n_{G}$ is the optimal working point, which is to be adjusted constantly during operation with a DC "feedback" gate (see Fig. 2a).

\section{B. Microwave response $\partial_{C_{Q}} \nu_{r}$ and $\left|\partial_{\nu} S_{21}\right|$}

The second term entering Eq. (3) is written $\partial_{C_{Q}} \nu_{r}=$ $-\nu_{r} / 2 C_{r}$, assuming that the $\mu \mathrm{w}$ voltage across the resonator capacitor appears fully on the $\mu \mathrm{w}$ gate of the CPB 
(i.e. that the CPB is effectively placed in parallel with the capacitance of the resonator).

To obtain $\left|\partial_{\nu} S_{21}\right|$, we first express the complex transmission coefficient $S_{21}$ (see Fig. 2,b) as a function of the reduced frequencies $x=\nu / \nu_{r}-\nu_{r} / \nu \approx \nu / \nu_{r}-1$ and $y=2 Q_{c} x$. In the side-coupled geometry

$$
S_{21}(x)=1-\frac{1}{1+Q_{c} / Q_{i}+2 i Q_{c} x}=\frac{1}{1+\frac{1}{r+i y}},
$$

where $Q_{i}$ and $Q_{c}$ are the resonator quality factors due to internal losses and coupling to the $50 \Omega$ transmission line, respectively, and $r=Q_{c} / Q_{i}$.

The internal quality factor can be expressed as $Q_{i}^{-1}=$ $\left(\kappa_{\mathrm{TLS}}+\kappa_{\text {rad }}+\kappa_{\text {diss }}+\kappa_{\text {mag }}+\kappa_{\text {ports }}\right) / \omega_{r}$, taking into account the energy decay rates due to dielectric two level systems (TLS) 25, radiation in free space (rad), Joule dissipation (diss), magnetic field induced losses (mag), or losses towards other electrodes coupled to the resonator (e.g., the gates connected to the $\mathrm{CPB}$ ).

The coupling quality factor $Q_{c}$ is given by the mutual inductance $M_{c}$ and/or coupling capacitance $C_{c}$ to the transmission line. For weak coupling we have $Q_{c} \approx$ $\left|Z_{\text {coupler }}\right|^{2} / Z_{\text {line }} Z_{r}$ (resp. $Q_{c} \approx Z_{\text {line }} Z_{r} /\left|Z_{\text {coupler }}\right|^{2}$ ) in the case of a purely capacitive (resp. inductive) coupling, with $Z_{\text {coupler }}=1 / j C_{c} \omega_{r}$ (resp. $j M_{c} \omega_{r}$ ) the impedance of the coupling element at the resonator frequency $\omega_{r}$, and $Z_{\text {line }}=Z_{0} / 2$ the line impedance as seen from the coupling point. For a distributed coupling, this generalizes to $Q_{c} \approx \omega_{c}^{2} / \omega_{0}^{2}$, with $\omega_{c}^{2}=1 / M_{c} C_{c}$, with values of $C_{c}$ and/or $M_{c}$ that are usually deduced from numerical simulations.

It is also useful to define the total quality factor $Q_{t}^{-1}=$ $Q_{c}^{-1}+Q_{i}^{-1}$ that relates the power $P_{\text {in }}$ injected at the input port 1 of the transmission line (Fig. 2) to the average number of photons $\bar{N}$ inside the resonator:

$$
\bar{N}=\frac{P_{\text {in }}}{\hbar \omega_{r}^{2}}\left[\frac{Z_{0}}{Z_{r}}+\frac{2 Q_{t}}{(1+r)}\right] \approx \frac{P_{\text {in }}}{\hbar \omega_{r}^{2}} \frac{2 Q_{t}}{(1+r)} \quad \text { for } Q_{t} \gg 1 .
$$

The modulus of the $S_{21}$ variations with respect to a change in the resonance frequency,

$$
\left|\partial_{\nu} S_{21}\right|=\frac{1}{\nu_{r}} \frac{2 r Q_{i}}{(1+r)^{2}+y^{2}} \quad[V / \mathrm{Hz} / \text { input } V],
$$

has a maximum at the resonance frequency $(y=0)$ and increases with $Q_{i}$ (see Fig. 2 c). Given some unavoidable internal losses (onto which one has limited control), the best resonator linear responsivity is achieved at critical coupling $Q_{c}=Q_{i}(r=1)$. However we will see in section IIIE2 that the result is different when taking the nonlinearity into account.

\section{Linear sensitivity limited by the amplification chain}

The detection sensitivity is now determined by the noise sources. The main and only sample-independent one comes from the $\mu \mathrm{w}$ amplification chain, the noise of which is always dominated by the first amplification stage. In this section where we consider only linear behaviours of all the components, the first amplification stage is a high electron mobility transistor (HEMT) amplifier located at $4 \mathrm{~K}$. Typical commercially available HEMT amplifiers have noise temperatures around $T_{N}=$ $4 K$ in the frequency range of interest $([1-5] \mathrm{GHz})$, corresponding to a noise power $k_{B} T_{N}$ per unit bandwidth. By definition, the sensitivity $S_{e}$ of the electrometer in $e^{-} / \sqrt{\mathrm{Hz}}$ is the charge variation inducing a power variation at the amplifier input equal to $k_{B} T_{N} \times 1 \mathrm{~Hz}$ (signal to noise ratio of 1 in a bandwidth of $1 \mathrm{~Hz}$ ). It is thus the voltage noise spectral density $\sqrt{k_{B} T_{N} Z_{0}}$ divided by the electrometer responsivity, that is

$$
S_{e}=\sqrt{k_{B} T_{N} /\left(P_{\text {in }}\left|\partial_{n_{G}} S_{21}\right|^{2} / 4\right)}
$$

with $P_{\text {in }}$ the power at the sample input. Combining Eq. (4) and (6) into Eq. (3) yields the setup limited charge sensitivity in the linear regime at low $\mu \mathrm{w}$ power and optimal $n_{G}$ :

$$
\begin{aligned}
& S_{e}^{\mathrm{lin}}=c_{0} \sqrt{\frac{k_{B} T_{N}}{P_{\mathrm{in}}}} \frac{(1+r)}{Q_{t}} \frac{C_{r}}{\eta_{G, r} C_{G, r}}\left(\frac{E_{J}}{E_{C}}\right)^{2} \\
& =\frac{c_{0}}{4} \sqrt{\frac{k_{B} T_{N}}{P_{\mathrm{in}}}} \frac{(1+r)}{Q_{t}}\left(\frac{E_{J}}{\hbar g}\right)^{2} \frac{\hbar \omega_{r}}{E_{C}} \quad\left[e^{-} / \sqrt{\mathrm{Hz}}\right] \text {, }
\end{aligned}
$$

where $c_{0}=25 \sqrt{5} / 48 \simeq 1$ and $\hbar g=\sqrt{\frac{\eta_{G, r} C_{G, r}}{4 C_{r}}} \sqrt{\hbar \omega_{r} E_{C}}$ expresses the capacitive coupling between the CPB and the resonator in terms of energy (or frequency), as detailed further in section IIIE 1.

Optimizing our electrometer consists in minimizing $S_{e}^{\text {lin }}$ by adjusting all independent parameters. At first glance, parameters in (8) could seem independent, though it is not the case. $P_{\text {in }}$ is constrained below a maximum value due to two nonlinearities: the nonlinearity of the quantum capacitance response $\left(\partial_{n_{G}} C_{Q, \mu}\right)$ presented in the next section, and the nonlinearity of the resonator response $\left(\left|\partial_{\nu} S_{21}\right|\right)$ inherited from its coupling to the $\mathrm{CPB}$, treated in section IIIE1.

\section{Smoothing of the quantum capacitance by the microwave signal}

Since the CPB's quantum capacitance is nonlinear in $n_{G}$, the $\mu \mathrm{w}$ gate charge $\delta n_{G}=$ $\eta_{G, r} \sqrt{\pi Z_{r} / R_{Q}} \sqrt{\bar{N}} \hbar \omega_{r} / E_{C}$, with $R_{Q}=h / 4 e^{2}$ the superconducting quantum of impedance, has to be small compared to the period $\left(\delta n_{G} \ll 1\right)$. Otherwise, the sharp $C_{Q}$ variations around $n_{G}^{\text {opt }}$ responsible for the high sensitivity of the electrometer (see Fig. 11. c) is smoothed and the sensitivity degraded. This smoothing is calculated here in a semi-classical way. We define the average capacitance

$$
\overline{C_{Q, r}}=\frac{1}{2 \pi} \int_{0}^{2 \pi} C_{Q, r}\left(n_{G, \mathrm{dc}}+\frac{\delta n_{G}}{2} \cos \theta\right) d \theta
$$




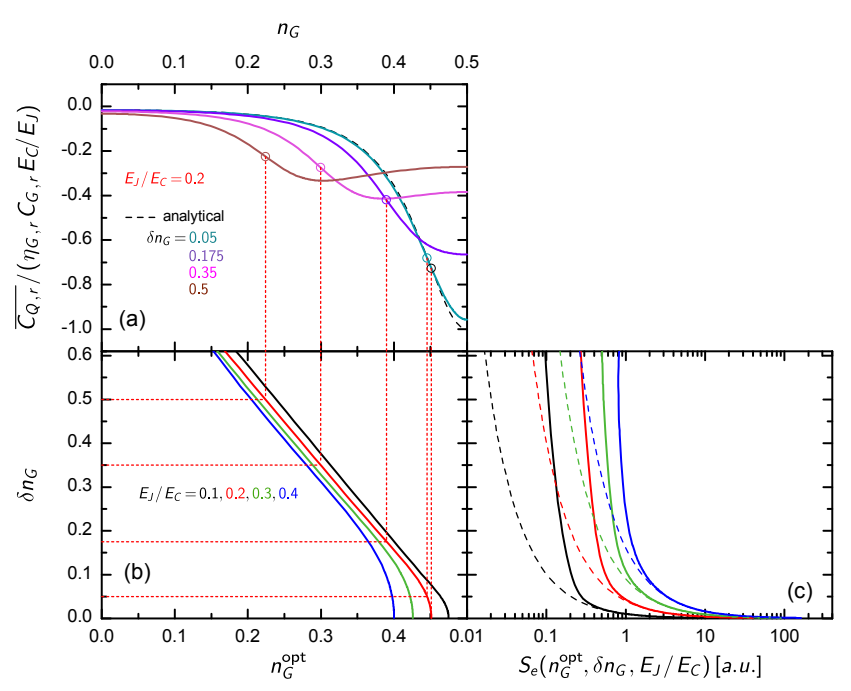

Figure 3. Smoothing of $C_{Q}$ with $\mu$ w probe amplitude $\left(\propto \delta n_{G}\right)$ and degradation of sensitivity $S_{e}$. (a) Smoothed quantum capacitance $\overline{C_{Q, r}}$ as a function of $n_{G}$ for $E_{J} / E_{C}=0.2$ and several $\mu \mathrm{w}$ excitations $\delta n_{G}$. Dots indicate the resulting optimal bias $n_{G}^{\text {opt }}\left(\delta n_{G}\right)$, at which charge responsivity $\partial_{n_{G}} C_{Q, r}$ is maximum. (b) Variation of $n_{G}^{\text {opt }}$ with $\delta n_{G}$ (swapped axes) at four values of $E_{J} / E_{C}$. (c) Sensitivity $S_{e}$ versus $\mu \mathrm{w}$ amplitude $\delta n_{G}$ at $n_{G}^{\mathrm{opt}}\left(\delta n_{G}\right)$ (continuous lines), degraded compared to the linear sensitivity (dashed lines) of Eq. (8).

probed by the $\mu \mathrm{w}$ tone as the integral of the quantum capacitance over a period of the microwave, assuming a linear resonator response.

Figure 3, a shows the result of this averaging for several values of the $\mu \mathrm{w}$ excitation $\delta n_{G} \lesssim 1 / 2$. Note that the optimal working point $n_{G}^{\text {opt }}$ now depends on power $P_{\text {in }}$ and has to be computed numerically (Fig. 3.b). The resulting $\mu \mathrm{w}$ averaged sensitivity $S_{e}\left(n_{G}^{\mathrm{opt}}\right)$ degrades at high $\delta n_{G}$ as shown in Fig. 3 c. This degradation can be kept below a factor two by limiting $\delta n_{G}$ below 0.1 .

\section{E. Nonlinearity of the resonator response}

\section{The CPB-resonator Hamiltonian}

Up to now we have forgotten the nonlinearity of the resonator inherited from the very nonlinear CPB device coupled to it. We will show that this effect can be already important for only a few photons in the resonator and that it modifies strongly the sensitivity dependence (8) on input power, quality factors and resonator-CPB coupling. This nonlinearity is calculated below with a full quantum treatment of the CPB-resonator system.

In the circuit quantum electrodynamics language, the total Hamiltonian of the system reads [26, 27]

$$
H_{\mathrm{tot}}=H_{r}+H_{\mathrm{CPB}}+H_{c}
$$

with

$$
\begin{aligned}
& H_{r}=\hbar \omega_{r}\left(a^{\dagger} a+\frac{1}{2}\right), \\
& H_{\mathrm{CPB}}=\sum_{k} \varepsilon_{k}|k\rangle\langle k|, \\
& H_{c}=2 \hbar g \hat{n}\left(a+a^{\dagger}\right),
\end{aligned}
$$

where $a$ and $a^{\dagger}$ are the photon annihilation and creation operators of the bare resonator, and $\hbar g=e V_{\mathrm{rms}} C_{G, r} / C_{\Sigma}$ is the CPB-resonator coupling factor with $V_{\mathrm{rms}}=$ $\sqrt{\hbar \omega_{r} / 2 C_{r}}$ the rms voltage on the $\mu \mathrm{w}$ gate of the CPB produced by zero point fluctuations. Here, the field amplitude $\left(a+a^{\dagger}\right)$ in the coupling term reflects the $\mu \mathrm{w}$ gate charge amplitude $\delta n_{G}=\sqrt{\bar{N}} 2 \hbar g / E_{C}$ entering the CPB's charge term $E_{C}\left(\hat{n}-n_{G}\right)^{2}$ in Eq. (1).

Expressing the operator $\hat{n}=\sum_{k, l}\langle k|\hat{n}| l\rangle|k\rangle\langle l|$
in
the $\langle k|\hat{n}| l\rangle=-i \int_{-\pi}^{\pi} d \theta \varphi_{k}^{*}(\theta) \partial_{\theta} \varphi_{l}(\theta)$ and $\varphi_{k}(\theta)=\langle\theta \mid k\rangle$, where $\varphi_{k}(\theta)$ is the $\mathrm{CPB}$ wavefunction in the conjugate phase representation [16], allows us to diagonalize $H_{\text {tot }}$ exactly in the tensorial product of the two eigenbases.

Since our $\mathrm{CPB}$ is operated in the charge regime $E_{J} / E_{C} \ll 1$ at $n_{G}^{\text {opt }}$ close to $1 / 2$, one could think that it is modeled sufficiently accurately by the two level system (TLS) made of the $\{|n=0\rangle,|n=1\rangle\}$ charge states at $n_{G}=1 / 2$. In this approximation $H_{\text {tot }}$ takes the JaynesCumings form (eq. 16 in [26] with $\theta=\pi / 2$ )

$$
H_{\mathrm{JC}}=\hbar \omega_{r}\left(a^{\dagger} a+\frac{1}{2}\right)+\frac{\hbar \Omega}{2} \sigma_{Z}+\hbar g\left(\sigma^{+}+\sigma^{-}\right)\left(a+a^{\dagger}\right),
$$

with $\hbar \Omega \equiv \varepsilon_{1}-\varepsilon_{0}$ the TLS transition energy, and $\sigma^{+}$, $\sigma^{-}$and $\sigma_{Z}$ the TLS raising, lowering, and z Pauli operators, respectively. Neglecting the fast oscillating terms $\sigma^{+} a^{\dagger}$ and $\sigma^{-} a$ that do not conserve the number of excitations and taking into account that the CPB transition and the resonator frequencies are very different $\left(\Delta \equiv \Omega-\omega_{r} \gg g\right), H_{\mathrm{JC}}$ can be solved analytically in the so-called rotating wave approximation (RWA), which yield a dispersive hamiltonian [27]

$H_{\text {disp }}=\hbar\left(\omega_{r}+\chi \sigma_{z}\right) a^{\dagger} a+\hbar(\Omega-\chi) \sigma^{+} \sigma^{-}+\frac{\hbar K_{\mathrm{TLS}}}{2} a^{\dagger 2} a^{2}$

exhibiting a CPB state-dependent resonator shift $\mp \chi=$ $\mp g^{2} / \Delta$ and a Kerr nonlinearity per photon [28]

$$
K_{\mathrm{TLS}}=2 \Delta(g / \Delta)^{4} .
$$

However, within the relevant parameter window for electrometry, solving numerically $H_{\text {tot }}$ (or $H_{\mathrm{JC}}$ keeping all terms) yields very different results from those obtained for $H_{\text {disp }}$ in the RWA, the nonlinearity at low photon numbers being for instance three times larger.

Consequently, we solve Eq. (99) numerically by truncating the $\mathrm{CPB}$ and resonator Hilbert spaces to three eigenstates and $N_{\text {tot }}=200$ Fock states, respectively [29]. From the eigenspectrum, we select the energies $E_{0, N}$ of the eigenstates $|0, N\rangle$ corresponding to the CPB being mostly in its ground state $|0\rangle$ hybridized with the 
resonator mostly in Fock state $|N\rangle$. We then plot in Fig. 4 the transitions frequencies $\Delta \omega_{N, \text { rel }} \equiv\left(\omega_{N}-\right.$ $\left.\omega_{r}\right) / \omega_{r}=\left(E_{0, N+1}-E_{0, N}\right) / \hbar \omega_{r}-1$ between two successive $|N\rangle$ states, relative to the bare resonator frequency $\omega_{r}$, together with the results obtained within the RWA (dashed lines).

The dependence on $n_{G}$ is shown on Fig. 4.a for two pairs of $\left\{E_{J}, g\right\}$ values. The resonator nonlinearity shows up as a dispersion of $\Delta \omega_{N \text {,rel }}$ with Fock index $N$, with a maximum spread at $n_{G}=1 / 2$. This shift of $\Delta \omega_{N \text {,rel with }}$ $N$ is plotted in Fig. 4 b-d (swapped axis) at $n_{G}^{\text {opt }}$ for different sets of parameters $\left\{\omega_{r}, E_{J}, g\right\}$. The spectrum and its dependence on $N$ differ significantly from the RWA prediction, as seen by comparing the dashed lines with the solid lines in insets a and d of Fig. 4. Besides, we also observe peaks at certain $N$ values, corresponding to resonant transitions $|0, N>\leftrightharpoons| 1, N-m>$ involving a coherent exchange of $m$ photons. Their existence depends on the coupling strength $g$ as shown in fig. $4 \mathrm{~d}$, and the specific $N$ at which they occur depends on $\omega_{r}$ and $E_{J}$ (on the commensurability of the $|0, N\rangle$ and $|1, N\rangle$ ladders), as shown in Fig. 4 b. Such resonance have of course to be avoided by design for our electrometry application.

These results illustrate how the RWA fails to describe our system, in particular when $g$ becomes non negligible with respect to $\omega_{r}$, and show that the full complexity of the Hamiltonian has to be taken into account to design properly a $\mu \mathrm{w}-\mathrm{CPB}$ electrometer.

\section{Nonlinear corrections and boost of the charge sensitivity $S_{e}$}

To incorporate easily the nonlinearity computed above in our calculation of the charge sensitivity, and although this nonlinearity slightly departs from a pure Kerr behavior, we choose to approximate it by a Kerr nonlinearity with the Kerr constant $K_{0}$ that linearizes our numerical results at small $N: \omega_{N}=\omega_{0}+N K_{0}$.

We now calculate numerically the nonlinear corrections to $\left|\partial_{\nu} S_{21}\right|$ entering the responsivity $\left|\partial_{n_{G}} S_{21}\right|$ in Eq. (3), and how they affect the sensitivity $S_{e}$ in Eq. (8). We model the nonlinear resonator by the Hamiltonian

$$
H_{\mathrm{NL}}=\hbar \omega_{0} a^{\dagger} a+\hbar \frac{K_{0}}{2} a^{\dagger 2} a^{2}
$$

with the new frequency $\omega_{0}$ and Kerr coefficient $K_{0}$ taken at $n_{G}^{\text {opt }}$. Following [30] with the convention $\beta_{\text {re }}=\beta e^{i \omega t}+$ c.c. for all the real fields $\beta_{\text {re }}$, the stationary equation of motion within the monochromatic approximation at the driving frequency $\omega$ reads

$$
\alpha\left[\frac{\kappa_{t}}{2}+i\left(\omega-\omega_{0}-K_{0}|\alpha|^{2}\right)\right]=i \alpha_{\mathrm{in}} \sqrt{\frac{\kappa_{c}}{2}},
$$

where $\kappa_{c}=\omega_{0} / Q_{C}, \kappa_{i}=\omega_{0} / Q_{i}$ and $\kappa_{t}=\kappa_{c}+\kappa_{i}$ are the modified coupling, internal, and total energy decay
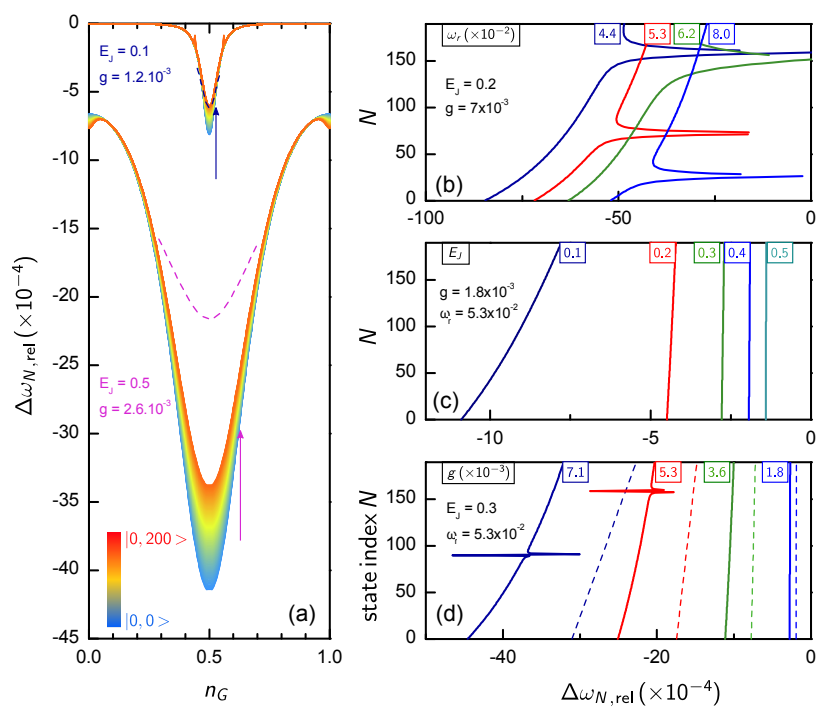

Figure 4. Resonator's relative transition frequencies $\Delta \omega_{N \text {,rel }}$ (the CPB being in its ground state) obtained by numerical diagonalization of the CPB-resonator Hamiltonian (solid lines), or using the Hamiltonian $H_{\text {disp }}$ (RWA - dashed lines). All parameters $E_{J}, \omega_{r}$, and $g$ are expressed in units of $E_{C}$. (a) Plot as a function of $n_{G}$ from $N=0$ to $N=200$ (color scale) for two pairs of $\left\{E_{J}, g\right\}$ values. The $n_{G}^{\text {opt }}$ positions are indicated by arrows. The RWA is plotted at $N=0$ only. (b,c,d) Plots as a function of the Fock state index $N$, at $n_{G}^{\text {opt }}$, and for different bare resonator frequencies (b), Josephson energies (c), and CPB-resonator couplings $g(\mathrm{~d})$, keeping all other parameters fixed.

rates, and $\alpha_{\text {in }}$ (resp. $\alpha$ ) is the reduced complex amplitude of the incident field from port 1 towards the resonator (resp. internal field inside the resonator), expressed in square root of photons per seconds (resp. square root of photons).

This third order polynomial equation has 1 or 3 real solutions depending on $\left|\alpha_{\text {in }}\right|$ and on the reduced frequency $\Omega \equiv 2\left(\omega-\omega_{0}\right) / \kappa_{t}$. The bifurcation from 1 to 3 solutions occurs at $\left\{\Omega^{B}=\sqrt{3},\left|\alpha_{\text {in }}^{B}\right|^{2}=\kappa_{t}^{3} /\left(3 \sqrt{3} \kappa_{c}\left|K_{0}\right|\right)\right\}$. The internal amplitudes are plotted on Fig. 5 a as a function of $\Omega$ for various input powers $P_{\text {in }}=\left|\alpha_{\text {in }}\right|^{2} \hbar \omega_{0}$. Above bifurcation, one of the solutions is metastable, and the internal field is hysteretic (arrows on the figure) and cannot be exploited for continuous detection. Our electrometer will thus be operated with the resonator below bifurcation.

Then, the output microwave field

$$
\alpha_{\mathrm{out}} \simeq \alpha_{\mathrm{in}}+i \sqrt{\frac{\kappa_{c}}{2}} \alpha
$$

on port 2 results from the interference between the incident field and the field re-emitted by the resonator to the right direction, which yields now an $\alpha$-dependent transmission coefficient

$$
S_{21}=1-\frac{\kappa_{c}}{\kappa_{t}+2 i\left(\omega-\omega_{0}-K_{0}|\alpha|^{2}\right)} .
$$



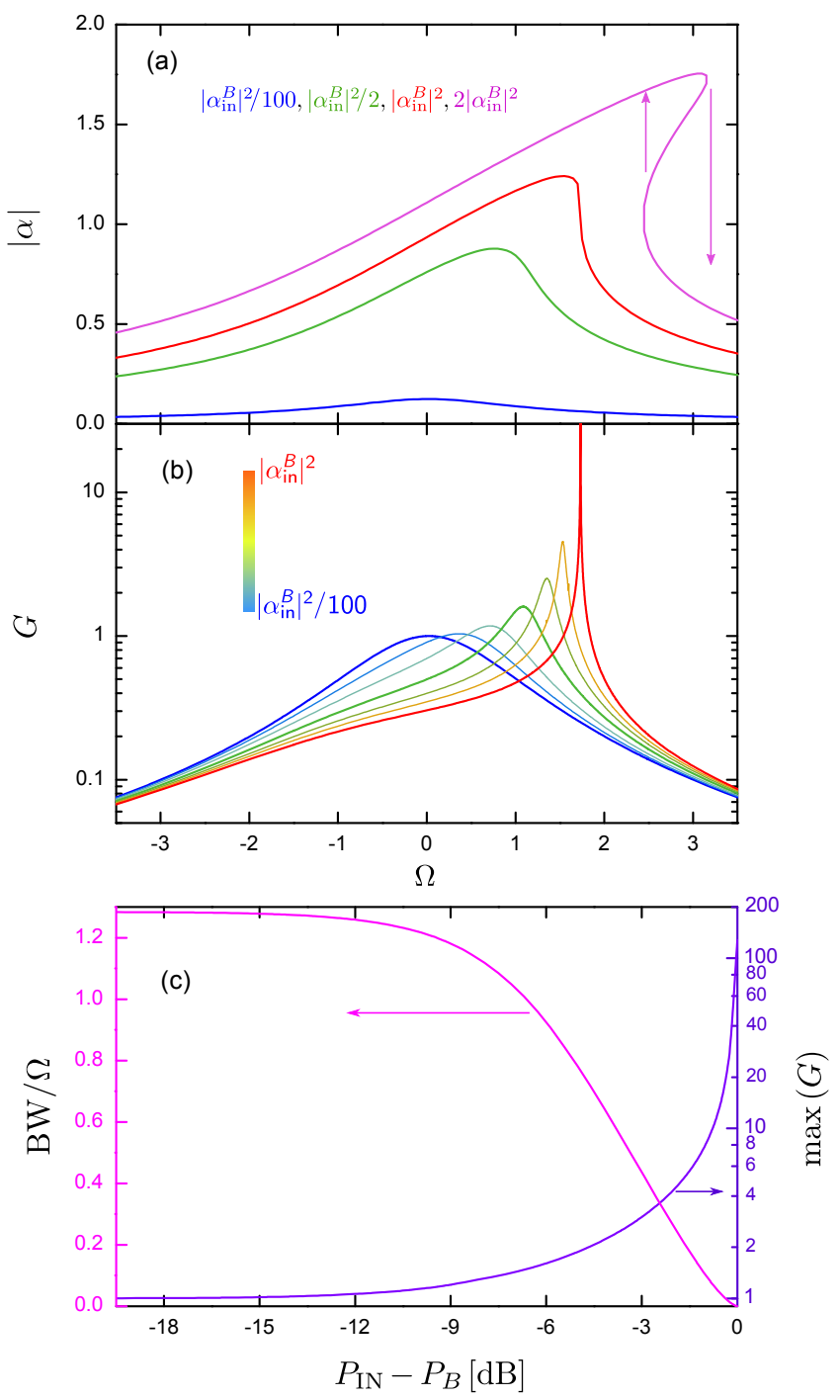

Figure 5. Non linear dynamics of the Kerr resonator in the side-coupled geometry as a function of the input power. (a) Intra-resonator field amplitude $|\alpha|$ as a function of reduced frequency $\Omega$ for several input photons rates $\left|\alpha_{\text {in }}\right|^{2}$ below and above bifurcation. (b) Responsivity $\left|\partial_{\nu} S_{21}\right|$ versus frequency $\Omega$ at several $\left|\alpha_{\text {in }}\right|^{2}$, from the linear regime (blue curve) to the bifurcation (red curve), normalized to the linear responsivity at $\Omega=0$. (c) Kerr resonator gain and gain bandwidth in $\Omega$ units versus input power relative to bifurcation power.

The corresponding responsivity normalized to Eq. (6) for a comparison with the linear case, $G=$ $\left|\partial_{\nu} S_{21}\right| /\left|\partial_{\nu} S_{21}\left(\alpha_{\text {in }}=0\right)\right|$, is plotted in Fig. 5 b as a function of frequency for several input powers up to the bifurcation. As bifurcation is approached, an enhancement of the responsivity is clearly observed $(G>1)$, while the responsivity bandwidth is reduced [31. The gain in responsivity as well as its corresponding -3dB gain bandwidth BW are plotted on Fig. 5.c as a function of the incident power.

An interesting property of this non linear resonator is that it lowers the effective noise temperature of the amplification chain. Indeed, with a frequency above $4 \mathrm{GHz}$, the superconducting resonator operated in a dilution refrigerator at a temperature of about $20 \mathrm{mK}$ is cold enough to be in the quantum regime with very little photon noise. More quantitatively, its noise temperature $T_{N, r}=\hbar \omega_{r} / 2 k_{B}$ is much lower than that of the following HEMT amplifier, and the effective noise temperature of the amplification chain becomes $T_{N}=$ $T_{N, r}+T_{N, \text { HEMT }} / G^{2}$. As the microwave power $P_{\text {in }}$ and consequently $\delta n_{G, \mu}=2 \hbar g \alpha / E_{C}$ are increased up to bifurcation, $T_{N}$ decreases as shown in fig. 6. a which correlatively improves the sensitivity. By noting that the dependence of $G$ on $P_{\text {in }}-P_{B}$ is universal we can infer that the sensitivity has a universal dependence on $\delta n_{G}$ (or $\bar{N}$ ) upon approaching the bifurcation, which we show on Fig. 6, b.

Consequently, we choose to operate our electrometer at an input power $P_{\text {in }} \sim P_{B}$ close to bifurcation. We also choose the $n_{G}^{\text {opt }}$ working point at which the slope $\partial_{q} C_{Q}$ is negative such that a detected charge $q$ will displace $n_{G}$ further away from $1 / 2$, lowering $K_{0}$ and thus moving the system further away from bifurcation. Assuming a large quality factor $Q_{t}$, we thus obtain a quantum limited charge sensitivity at bifurcation

$S_{e}^{B}\left(\omega_{r}, E_{J}, E_{C}, g, P_{B}\right)=\frac{c_{0}}{4 \sqrt{2}} \frac{(1+r)}{Q_{t}} \frac{\hbar \omega_{r}}{E_{C}}\left(\frac{E_{J}}{\hbar g}\right)^{2} \sqrt{\frac{\hbar \omega_{r}}{P_{B}}}$

with $P_{B}=\hbar(1+r) \omega_{r}^{3} /\left(3 \sqrt{3} Q_{t}^{2}\left|K_{0}\right|\right)$.

The remaining parameter to expand is $K_{0}$. To do so we use the RWA as an analytical guideline, even though we have shown in section IIIE1 that this simplification does not give quantitative results. Using $K_{\text {TLS }}$ from Eq. 10 for $K_{0}$ yields $P_{B} \propto g^{-4}$ and a much simpler form for the sensitivity,

$$
\begin{aligned}
S_{e, \mathrm{RWA}}^{B}\left(\frac{\hbar \omega_{r}}{E_{J}}, \frac{E_{J}}{E_{C}}, E_{C}\right) \\
\quad \approx \sqrt{\frac{1+r}{2}}\left(1-\frac{\hbar \omega_{r}}{E_{J}}\right)^{-3 / 2} \sqrt{\frac{E_{J}}{E_{C}}} \sqrt{\frac{\hbar}{E_{C}}},
\end{aligned}
$$

which strikingly no longer depends on $g$ and $P_{B}$. This can be understood in the following way: as $g$ increases, better responsivity is achieved while at the same time the highest allowed working power decreases due to nonlinearity, hence lowering the signal to noise ratio; those two effects compensate exactly within the RWA.

We then check numerically that the independence of $S_{e}^{B}$ on $g$ and $P_{B}$ remains true within a few percents for the exact Hamiltonian $(9)$ and for all parameter sets satisfying $g / \omega_{r} \in[0.008-0.2]$, provided $m$-photons resonant exchange processes are avoided. This means that the sensitivity at bifurcation takes the reduced form $s_{e}^{B}=S_{e}^{B} /\left(\sqrt{(1+r) \hbar / 2 E_{C}}\right)$ inspired from Eq. 12, 

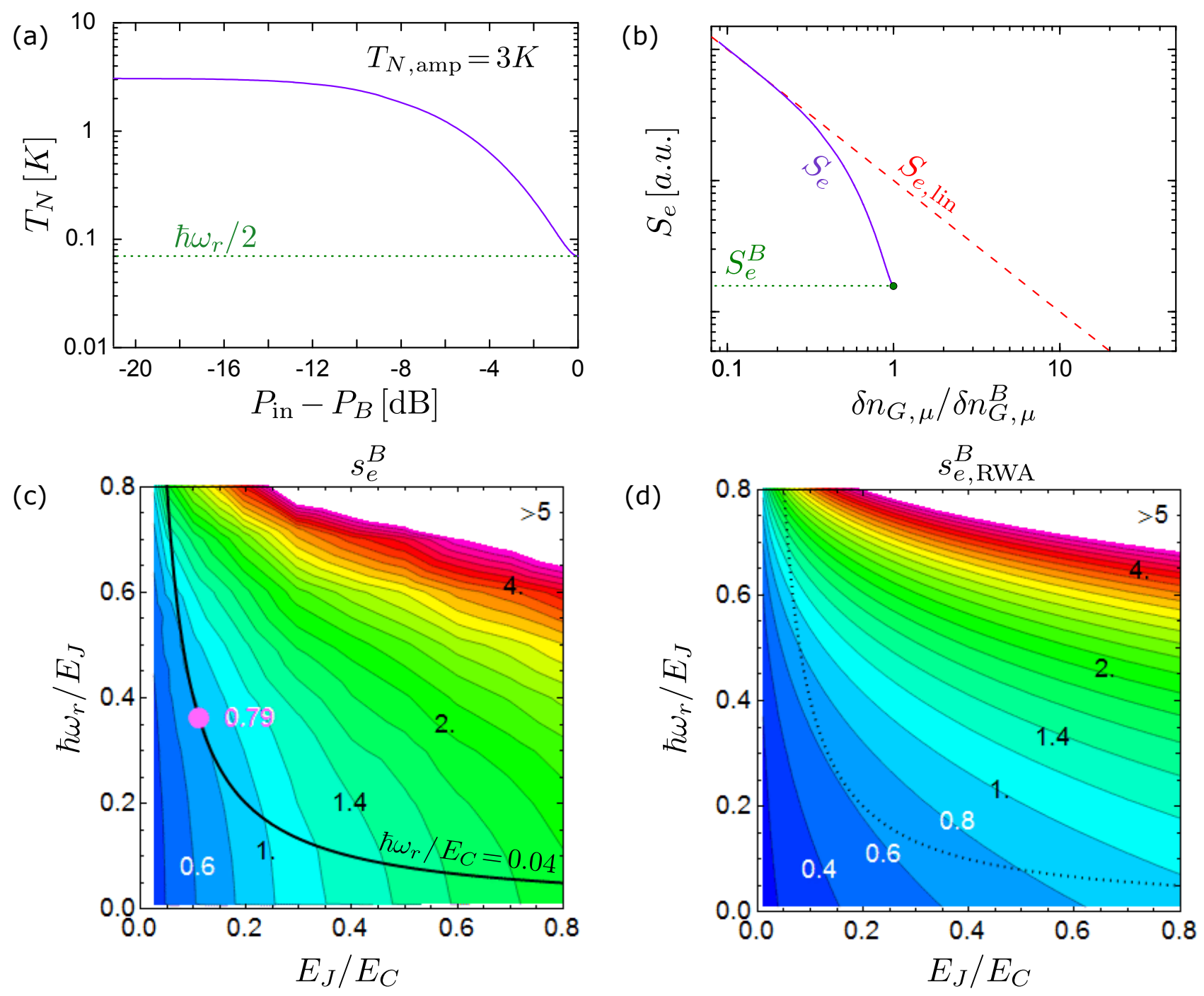

Figure 6. Consequences of parametric amplification on the electrometer sensitivity, and optimal choice of parameters assuming a noise temperature $T_{N}=3 K$ for the HEMT. (a) Effective noise temperature versus input power up to bifurcation. The lower bound of $T_{N}$ reached at bifurcation is given by the quantum limit, i.e. $\hbar \omega_{r} / 2 k_{B} \approx 70 \mathrm{mK}$ for a $4 \mathrm{GHz}$ resonator. (b) Reduced

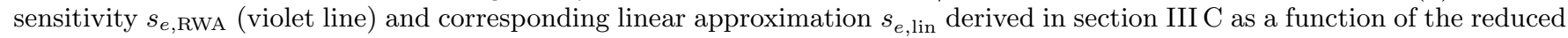
$\mu \mathrm{w}$ gate charge relative to bifurcation. c) Exact reduced sensitivities $s_{e}^{B}$ as a function of $E_{J} / E_{C}$ and $\hbar \omega_{r} / E_{J}$. The solid line $\hbar \omega_{r} / E_{C}=0.04$ corresponds to the constraints $\hbar \omega_{r} / k_{B}=10 T=120 \mathrm{mK}$ and $E_{C} / k_{B}=3 K$. The magenta point indicates the best (minimum) sensitivity and fixes the remaining parameter $E_{J} / k_{B}=0.3 K$. d) Corresponding RWA approximation of the sensitivity $s_{e, \text { RWA. }}^{B}$

which depends only on $\hbar \omega_{r} / E_{J}$ and $E_{J} / E_{C}$, and that $E_{C}$ should be maximized.

We plot $s_{e}^{B}\left(\hbar \omega_{r} / E_{J}, E_{J} / E_{C}\right)$ in fig. 6. 6 as well as $s_{e, \mathrm{RWA}}^{B}$ in fig. 6. d for comparison. The reduced sensitivity $s_{e}^{B}$ is found to improve at low $\hbar \omega_{r} / E_{J}$ and low $E_{J} / E_{C}$. However the optimization is constrained: $E_{C}$ can be increased only up to a maximum value that depends on the technology used for the CPB fabrication, and $\omega_{r}$ cannot be lower than $5-10 k_{B} T / \hbar$ (with $T$ the base temperature of the electrometer) to keep the resonator in the quantum regime, which altogether defines a constant product for the remaining variables $\left(\hbar \omega_{r} / E_{J}\right) \times\left(E_{J} / E_{C}\right)$.
The optimal sensitivity is thus found at the minimum of $s_{e}^{B}$ along a hyperbola, as the one shown in fig. 6. b. for $\hbar \omega_{r} / k_{B}=0.12 K$ and $E_{C} / k_{B}=3 K$, i.e. values that can be reached in many laboratories. The corresponding optimum sensitivity is found on the graph at $E_{J} / k_{B}=0.3 \mathrm{~K}$.

To summarize, the sensitivity of the electrometer that we propose will be optimum when choosing a resonator having any characteristic impedance, the lowest frequency $\omega_{r}$ above $5-10 k_{B} T / \hbar$ and $Q$ factors $1000<$ $Q_{c} \ll Q_{i}(r \ll 1)$ contrary to what was found from Eq. (8), when maximizing the charging energy $E_{C}$, and operating at an input power $P_{\text {in }} \lesssim P_{B}$ slightly below the res- 
onator bifurcation whatever the CPB-resonator coupling $g$ is; the only remaining free parameter $E_{J}$ will finally be optimized according to the procedure described in fig. 6. c.

\section{OFFSET CHARGE NOISE AND QUASIPARTICLE POISONING}

We now discuss two problems often encountered when operating a CPB: offset gate charge noise of microscopic origin, and tunneling of residual quasiparticles in and out of the CPB island. We present briefly these issues and describe how to deal with them in practice in an actual electrometer.

\section{A. Offset gate charge noise}

Offset charge refers to randomly fluctuating charged defects in the vicinity of the $\mathrm{CPB}$ island, which induce net gate charge variations (noise). Charge noise in CPBs has been found to come mainly from oxide defects either inside [32, 33 or close to 34, 35] the Josephson junction, that is from locations where the defects are more strongly coupled to the CPB electric dipole. The magnitude of charge noise can be reduced by improving the quality of the junction dielectric [36, though no definitive cure has been found yet and the subject is still an active field of research [37.

The spectral density of this noise is known to have a $1 / f^{\alpha}$ dependence [6] with $\alpha \bumpeq 1$, due to a collection of widely distributed two level systems [38, 39, which result in a continuous slow drift of $n_{G}$. This gives rise to two issues for the electrometer: (i) its sensitivity is degraded at low frequency due to charge noise, up to a frequency called the $1 / f$ corner, above which noise is dominated by $k_{B} T_{N}$ as discussed in section IIIE2, (ii) The sensitivity reaches its floor $S_{e}^{B}$ above the $1 / f$ corner, only on the condition that $n_{G}$ does not drift significantly away from $n_{G}^{\mathrm{opt}}$.

An efficient and easy way to compensate for the slow $n_{G}$ drift away from $n_{G}^{\text {opt }}$ is to adapt constantly the voltage applied to a feedback gate (see Fig. 2 and 7), in order to maintain a constant quantum capacitance, i.e. maintain the amplitude and phase of the microwave signal transmitted through the measuring line. This has both the advantages of keeping the optimal responsivity, and linearizing the electrometer response to a charge change.

The sensitivity degradation below the $1 / f$ corner can also be partially cured by coupling a number $N$ of identical CPB-resonator electrometers to the same device, which yields a $\sqrt{N}$ gain on sensitivity, assuming uncorrelated charge noises for the different CPBs. Note that the $\mu \mathrm{w}$ design proposed here conveniently enables frequency multiplexing of all the resonators by a single microwave line.

\section{B. Quasiparticle poisoning}

Although the volumic density $\rho_{\mathrm{qp}}$ of quasiparticle excitations in a superconductor should decrease with temperature $T$ as $\sim \exp \left(-\Delta / k_{B} T\right)$ with $\Delta$ the superconducting energy gap, one always observe at $\mathrm{mK}$ temperature outof-equilibrium quasiparticles at a much higher density, of the order of a few ones per $\mu m^{3}$ [40. These quasiparticles can tunnel across the junction and poison the island with an unpaired electron. As a consequence, the simple energy diagram of Fig. 1 $1 \mathrm{~b}$ valid for the so-called "even" states corresponding to all electrons paired, becomes the diagram of Fig. $7 \mathrm{a}$ with an additional "odd" ground state band shifted by $1 e$ in $n_{G}$ and by the free-energy difference $\Delta F$ [1]. Whenever a quasiparticle tunnels, the state of the island switches between the two bands of different parities [11, which switches on and off the quantum capacitance at $n_{G}^{\mathrm{opt}} \simeq 1 / 2$. With the corresponding switching rates $\Gamma_{o \rightarrow e}$ and $\Gamma_{e \rightarrow o}$, the CPB spends a fraction of the time $p_{e}=\Gamma_{o \rightarrow e} /\left(\Gamma_{o \rightarrow e}+\Gamma_{e \rightarrow o}\right)$ in the good (i.e. charge sensitive) even state.

The thermodynamics and the kinetics [9, 41, 42] of these jumps are governed in particular by $\rho_{\mathrm{qp}}$, and by the density $\rho_{\mathrm{sg}}$ of island subgap states. Using an island material so that $\rho_{\mathrm{sg}}$ is small, one can make $\Gamma_{e \rightarrow o}$ very slow at low temperature, as demonstrated with NbTiN islands and $\mathrm{Al}$ grounds 43, for which about 1 minute even state lifetime was observed. With such a lifetime much longer than the inverse bandwidth of the electrometer discussed in section III E 2, and in absence of specific feedback, the electrometer is operational only a fraction $p_{e}$ of the time, as illustrated in Fig. 7b. But it is also possible to program a feedback that shifts $n_{G}$ by $1 e$ whenever a poisoning event occurs, in order to actively restore the even state sensitivity.

\section{INTEGRATING OUR ELECTROMETER IN A MACROSCOPIC DETECTOR}

The range of applications of the electrometer proposed here is wide: it could be used to study new quantum electrical devices or address present-day questions in mesoscopic physics, like measuring the charge of Majorana split states. However our goal is to use it for detection of rare astroparticle events creating a bunch of charges inside a massive semiconducting cristal (e.g Germanium) placed at a few $\mathrm{mK}$.

The main problem in this bolometric application is the small coupling between the massive block and the mesoscopic electrometer: indeed, the block having a large selfcapacitance compared to the CPB gate, only a small frac- 


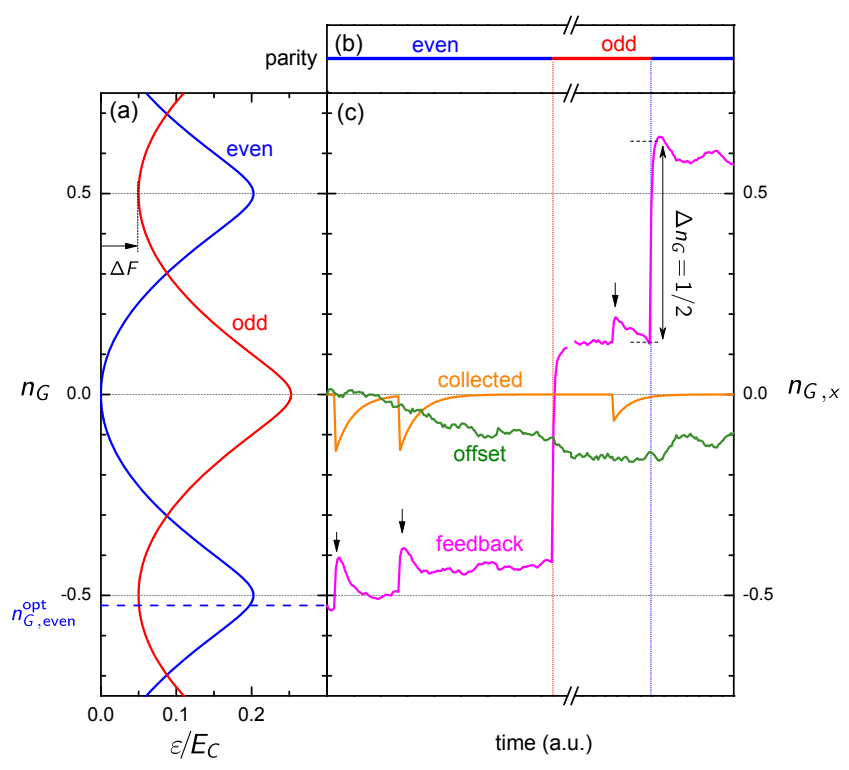

Figure 7. Sketch of a measurement in feedback mode, in presence of parity jumps and $1 / f$ charge noise. (a) Even and odd ground state energy bands of the CPB as a function of total gate charge $n_{G}$ at $E_{J} / E_{C}=0.1$ (swapped axes). (b,c) Sketch of a possible time variation of the island parity, offset charge drift, and collected charge on the detector gate. Corresponding feedback gate charge maintaining the amplitude and phase of the transmitted microwave signal, calculated with a finite feedback speed higher than the detector discharge speed. The three detected events are indicated by arrows. The detector is blind during the recovery time after every parity jump.

tion $\left(\sim 10^{-5}\right)$ of the total created charge is collected onto the CPB gate. To maximize the coupling, the detector gate capacitance between the CPB island and the semiconducting block should be made large, although we have concluded from our optimization that $E_{C}$ should be maximized. This means that the total capacitance budget is tight, and that a maximum amount of this budget should be devoted to coupling to the semiconducting block.

A simple evaluation of this budget goes as follows: The total capacitance is distributed between a Josephson junction of $100 \times 100 \mathrm{~nm}\left(C_{J} \sim 500 \mathrm{aF}\right)$, a detector gate of similar capacitance $\left(C_{G, d} \sim 500 \mathrm{aF}\right)$, and the other gates and parasitic capacitances $\left(C_{G, r}+C_{G, f} \sim 200 \mathrm{aF}\right)$, which results in a charging energy $E_{C} / k_{B}=3 K$. Taking a low-loss $(r=0.1)$ resonator of frequency $f_{r}=2.4 \mathrm{GHz}$, we find an optimum Josephson coupling $E_{J} / E_{C}=0.1$ and the corresponding quantum limited sensitivity $S_{e}^{B}=$ $9.10^{-7} e^{-} / \sqrt{\mathrm{Hz}}$, with a bandwidth determined by $\omega_{0} / Q_{t}$.

A Ge bolometer detector such as the ones used in CDMS 44 or EDELWEISS 45 can be optimized to have a capacitance between electrodes of about $C_{\mathrm{det}}=10 \mathrm{pF}$. Taking the capacitive division between $C_{\operatorname{det}}$ and $C_{G, d}$ into account yields an energy sensitivity of $1.8 \mathrm{eV} / \sqrt{\mathrm{Hz}}$ for neutral events, assuming an energy to charge conver- sion of $10 \mathrm{eV} / e^{-}$, typical for nuclear recoil in Germanium [46. This result outperforms by far (one to two orders of magnitude for similar $C_{\mathrm{det}}$ ) all existing semiconducting charge amplifiers, even when accounting for a larger capacitive division [4].

Now in terms of bare charge sensitivity of the electrometer alone, these $9.10^{-7} e^{-} / \sqrt{\mathrm{Hz}}$ could seem disapointing compared to the $\sim 10^{-6} e^{-} / \sqrt{\mathrm{Hz}}$ sensitivity reported for the best superconducting $\mu \mathrm{w}-\mathrm{CPB}$ or CPTs 8 . However, we stress that ours is given for a geometry suitable for coupling to macroscopic detectors, i.e. with a large coupling capacitance. Should we take the island geometrical parameters of ref. [8], i.e. an island capacitance $58 \mathrm{aF}$ $\left(E_{C} / k_{B}=64 K\right)$, we would predict a sensitivity one order of magnitude better $\left(S_{e}^{B}=0.3 \times \sqrt{(1+r) \hbar / 2 E_{C}} \simeq\right.$ $\left.0.8 \cdot 10^{-7} e^{-} / \sqrt{\mathrm{Hz}}\right)$, using the previous resonator parameters and the new optimum $E_{J}$ (along the hyperbola $\left.\hbar \omega_{r} / E_{C}=0.002\right)$.

\section{CONCLUSIONS}

We have shown how to optimize a wide-band $\mu$ wCooper Pair Box electrometer. By taking advantage of the resonator nonlinearity induced by the $\mathrm{CPB}$, a quantum limited sensitivity about ten times better than the linear one can be obtained, similar to what would be achieved using a separate quantum limited amplifier.

Interestingly, in the nonlinear case, the CPB-resonator coupling strength and the resonator impedance disappear from the optimization problem, which leads to a much simpler constrained numerical optimization.

Taking routinely achiveable parameters, we predict a conservative quantum limited sensitivity $S_{e}^{B}$ in the $10^{-7} e^{-} / \sqrt{\mathrm{Hz}}$ range, which should make possible to clarify the physics of new quantum electrical devices, and to fabricate much more sensitive bolometers for particle detection.

\section{ACKNOWLEDGEMENTS}

The authors are warmly thankful to D. Estève, P. Joyez and the Quantronics group for stimulating discussions about the CPB, and thank L. Dumoulin and S. Marnieros for sharing their expertise on bolometric dark matter detection. HLS aknowledges support from the FCS Campus Paris-Saclay under the project 2011-019T-COCA, from the ANR JCJC grant ANR-12-JS04-0007-01, and from the PNCG.

* Corresponding author: helene.le-sueur@csnsm.in2p3.fr 
[1] M. Field, C. G. Smith, M. Pepper, D. A. Ritchie, J. E. F. Frost, G. A. C. Jones, and D. G. Hasko. Measurements of Coulomb blockade with a noninvasive voltage probe. Physical Review Letters, 70(9):1311-1314, mar 1993.

[2] Gilad Ben-Shach, Arbel Haim, Ian Appelbaum, Yuval Oreg, Amir Yacoby, and Bertrand I. Halperin. Detecting Majorana modes in one-dimensional wires by charge sensing. Physical Review B, 91(4):45403, jan 2015.

[3] A. J. Anderson, J. M. Conrad, E. Figueroa-Feliciano, K. Scholberg, and J. Spitz. Coherent neutrino scattering in dark matter detectors. Physical Review D, 84(1):13008, jul 2011.

[4] D. Akimov, J. B. Albert, P. An, C. Awe, P. S. Barbeau, B. Becker, V. Belov, A. Brown, A. Bolozdynya, B. Cabrera-Palmer, M. Cervantes, J. I. Collar, R. J. Cooper, R. L. Cooper, C. Cuesta, D. J. Dean, J. A. Detwiler, A. Eberhardt, Y. Efremenko, S. R. Elliott, E. M. Erkela, L. Fabris, M. Febbraro, N. E. Fields, W. Fox, Z. Fu, A. Galindo-Uribarri, M. P. Green, M. Hai, M. R. Heath, S. Hedges, D. Hornback, T. W. Hossbach, E. B. Iverson, L. J. Kaufman, S. Ki, S. R. Klein, A. Khromov, A. Konovalov, M. Kremer, A. Kumpan, C. Leadbetter, L. Li, W. Lu, K. Mann, D. M. Markoff, K. Miller, H. Moreno, P. E. Mueller, J. Newby, J. L. Orrell, C. T. Overman, D. S. Parno, S. Penttila, G. Perumpilly, H. Ray, J. Raybern, D. Reyna, G. C. Rich, D. Rimal, D. Rudik, K. Scholberg, B. J. Scholz, G. Sinev, W. M. Snow, V. Sosnovtsev, A. Shakirov, S. Suchyta, B. Suh, R. Tayloe, R. T. Thornton, I. Tolstukhin, J. Vanderwerp, R. L. Varner, C. J. Virtue, Z. Wan, J. Yoo, C.H. Yu, A. Zawada, J. Zettlemoyer, A. M. Zderic, and Coherent Collaboration. Observation of coherent elastic neutrino-nucleus scattering. Science, page 0, aug 2017.

[5] EDELWEISS Collaboration, Q. Arnaud, E. Armengaud, C. Augier, A. Benot, L. Bergé, J. Billard, A. Broniatowski, P. Camus, A. Cazes, M. Chapellier, F. Charlieux, M. De Jésus, L. Dumoulin, K. Eitel, N. Foerster, J. Gascon, A. Giuliani, M. Gros, L. Hehn, Y. Jin, A. Juillard, M. Kleifges, V. Kozlov, H. Kraus, V. A. Kudryavtsev, H. Le-Sueur, R. Maisonobe, S. Marnieros, X.F. Navick, C. Nones, E. Olivieri, P. Pari, B. Paul, D. Poda, E. Queguiner, S. Rozov, V. Sanglard, S. Scorza, B. Siebenborn, L. Vagneron, M. Weber, and E. Yakushev. Optimizing EDELWEISS detectors for lowmass WIMP searches. ArXiv:1707.04308 [astro-ph, physics:physics], jul 2017.

[6] R. J. Schoelkopf, P. Wahlgren, A. A. Kozhevnikov, P. Delsing, and D. E. Prober. The Radio-Frequency Single-Electron Transistor (RF-SET): A Fast and Ultrasensitive Electrometer. Science, 280(5367):1238-1242, may 1998.

[7] Michel H. Devoret and Robert J. Schoelkopf. Amplifying quantum signals with the single-electron transistor. Nature, 406(6799):1039-1046, aug 2000.

[8] Henrik Brenning, Sergey Kafanov, Tim Duty, Sergey Kubatkin , and Per Delsing. An ultrasensitive radiofrequency single-electron transistor working up to $4.2 \mathrm{k}$. Journal of Applied Physics, 100(11):114321, dec 2006.

[9] O. Naaman and J. Aumentado. Time-domain measurements of quasiparticle tunneling rates in a single-CooperPair transistor. Physical Review B, 73(17):172504, may 2006.
[10] S. E. de Graaf, J. Leppäkangas, A. Adamyan, A. V. Danilov, T. Lindström, M. Fogelström, T. Bauch, G. Johansson, and S. E. Kubatkin. Charge Qubit Coupled to an Intense Microwave Electromagnetic Field in a Superconducting Nb Device: Evidence for PhotonAssisted Quasiparticle Tunneling. Physical Review Letters, 111(13):137002, sep 2013.

[11] P. Lafarge, P. Joyez, D. Esteve, C. Urbina, and M. H. Devoret. Measurement of the even-odd free-energy difference of an isolated superconductor. Physical Review Letters, 70(7):994-997, feb 1993.

[12] M. Büttiker. Zero-current persistent potential drop across small-capacitance Josephson junctions. Physical Review B, 36(7):3548-3555, sep 1987.

[13] V. Bouchiat, D. Vion, P. Joyez, D. Esteve, and M. H. Devoret. Quantum coherence with a single Cooper pair. Physica Scripta, 1998(T76):165, 1998.

[14] Y. Nakamura, Yu A. Pashkin , and J. S. Tsai. Coherent control of macroscopic quantum states in a singleCooper-Pair box. Nature, 398(6730):786-788, apr 1999.

[15] D. Vion, A. Aassime, A. Cottet, P. Joyez, H. Pothier, C. Urbina, D. Esteve, and M. H. Devoret. Manipulating the Quantum State of an Electrical Circuit. Science, 296(5569):886-889, may 2002.

[16] Audrey Cottet. Implementation of a Quantum Bit in a Superconducting Circuit. $\mathrm{PhD}$ thesis, Université Pierre et Marie Curie-Paris VI, 2002.

[17] A. Widom, G. Megaloudis, T. D. Clark, J. E. Mutton, R. J. Prance, and H. Prance. The Josephson pendulum as a nonlinear capacitor. Journal of Low Temperature Physics, 57(5-6):651-658, dec 1984.

[18] T. Duty, G. Johansson, K. Bladh, D. Gunnarsson, C. Wilson, and P. Delsing. Observation of Quantum Capacitance in the Cooper-Pair Transistor. Physical Review Letters, 95(20):206807, nov 2005.

[19] M. A. Sillanpää, T. Lehtinen, A. Paila, Yu. Makhlin, L. Roschier, and P. J. Hakonen. Direct Observation of Josephson Capacitance. Physical Review Letters, 95(20):206806, nov 2005.

[20] F. Persson, C. M. Wilson, M. Sandberg, and P. Delsing. Fast readout of a single Cooper-Pair box using its quantum capacitance. Physical Review B, 82(13):134533, oct 2010.

[21] T. R. Stevenson, F. A. Pellerano, and C. M. Stahle. Multiplexing of radio-frequency single-electron transistors. Applied Physics Letters, 80(16):3012-3014, apr 2002.

[22] Peter K. Day, Henry G. LeDuc, Benjamin A. Mazin, Anastasios Vayonakis, and Jonas Zmuidzinas. A broadband superconducting detector suitable for use in large arrays. Nature, 425(6960):817-821, oct 2003.

[23] K. D. Irwin and K. W. Lehnert. Microwave SQUID multiplexer. Applied Physics Letters, 85(11):2107-2109, sep 2004.

[24] V. Schmitt, X. Zhou, K. Juliusson, B. Royer, A. Blais, P. Bertet, D. Vion, and D. Esteve. Multiplexed readout of transmon qubits with Josephson bifurcation amplifiers. Physical Review A, 90(6):62333, dec 2014.

[25] John M. Martinis, K. B. Cooper, R. McDermott, Matthias Steffen, Markus Ansmann, K. D. Osborn, K. Cicak, Seongshik Oh, D. P. Pappas, R. W. Simmonds , and Clare C. Yu. Decoherence in Josephson Qubits from Dielectric Loss. Physical Review Letters, 95(21):210503, nov 2005. 
[26] Alexandre Blais, Ren-Shou Huang, Andreas Wallraff, S. M. Girvin, and R. J. Schoelkopf. Cavity quantum electrodynamics for superconducting electrical circuits: An architecture for quantum computation. Physical Review A, 69(6):62320, jun 2004.

[27] Jens Koch, Terri M. Yu, Jay Gambetta, A. A. Houck, D. I. Schuster, J. Majer, Alexandre Blais, M. H. Devoret, S. M. Girvin , and R. J. Schoelkopf. Charge-insensitive qubit design derived from the Cooper pair box. Physical Review A, 76(4):42319, oct 2007.

[28] Simon E. Nigg, Hanhee Paik, Brian Vlastakis, Gerhard Kirchmair, S. Shankar, Luigi Frunzio, M. H. Devoret, R. J. Schoelkopf, and S. M. Girvin. Black-Box Superconducting Circuit Quantization. Physical Review Letters, 108(24):240502, jun 2012.

[29] Note that the eigenenergies do not depend on this truncation for the CPB ground state and up to resonator level $N_{\text {tot }}-5$.

[30] B. Yurke and E. Buks. Performance of Cavity-Parametric Amplifiers, Employing Kerr Nonlinearites, in the Presence of Two-Photon Loss. Journal of Lightwave Technology, 24(12):5054-5066, dec 2006.

[31] R. Vijay, M. H. Devoret, and I. Siddiqi. Invited Review Article: The Josephson bifurcation amplifier. Review of Scientific Instruments, 80(11):111101, nov 2009.

[32] R. W. Simmonds, K. M. Lang, D. A. Hite, S. Nam, D. P. Pappas, and John M. Martinis. Decoherence in Josephson Phase Qubits from Junction Resonators. Physical Review Letters, 93(7):77003, aug 2004.

[33] Jürgen Lisenfeld, Grigorij J. Grabovskij, Clemens Müller, Jared H. Cole, Georg Weiss, and Alexey V. Ustinov. Observation of directly interacting coherent two-level systems in an amorphous material. Nature Communications, 6:6182, feb 2015 .

[34] O. Astafiev, Yu. A. Pashkin, Y. Nakamura, T. Yamamoto , and J. S. Tsai. Quantum Noise in the Josephson Charge Qubit. Physical Review Letters, 93(26):267007, dec 2004.

[35] G. Ithier, E. Collin, P. Joyez, P. J. Meeson, D. Vion, D. Esteve, F. Chiarello, A. Shnirman, Y. Makhlin, J. Schriefl, and G. Schön. Decoherence in a superconducting quantum bit circuit. Physical Review B, $72(13): 134519$, oct 2005.

[36] Seongshik Oh, Katarina Cicak, Jeffrey S. Kline, Mika A. Sillanpää, Kevin D. Osborn, Jed D. Whittaker, Raymond W. Simmonds, and David P. Pappas. Elimination of two level fluctuators in superconducting quantum bits by an epitaxial tunnel barrier. Physical Review B, 74(10):100502, sep 2006.

[37] A. Dunsworth, A. Megrant, C. Quintana, Zijun Chen, R. Barends, B. Burkett, B. Foxen, Yu Chen, B. Chiaro, A. Fowler, R. Graff, E. Jeffrey, J. Kelly, E. Lucero, J. Y. Mutus, M. Neeley, C. Neill, P. Roushan, D. Sank, A. Vainsencher, J. Wenner, T. C. White, and John M. Martinis. Characterization and Reduction of Capacitive Loss Induced by Sub-Micron Josephson Junction Fabrication in Superconducting Qubits. ArXiv:1706.00879 [quant-ph], jun 2017.

[38] Alexander Shnirman, Gerd Schön, Ivar Martin , and Yuriy Makhlin. Low- and High-Frequency Noise from Coherent Two-Level Systems. Physical Review Letters, 94(12):127002, apr 2005.

[39] Clemens Müller, Jared H. Cole , and Jürgen Lisenfeld. Towards understanding two-level-systems in amorphous solids - Insights from quantum devices.
ArXiv:1705.01108 [cond-mat, physics:quant-ph], may 2017.

[40] Anton Bespalov, Manuel Houzet, Julia S. Meyer , and Yuli V. Nazarov. Theoretical Model to Explain Excess of Quasiparticles in Superconductors. Physical Review Letters, 117(11):117002, sep 2016.

[41] R. M. Lutchyn and L. I. Glazman. Kinetics of quasiparticle trapping in a Cooper-Pair box. Physical Review B, 75(18):184520, may 2007.

[42] M. D. Shaw, R. M. Lutchyn, P. Delsing , and P. M. Echternach. Kinetics of nonequilibrium quasiparticle tunneling in superconducting charge qubits. Physical Review B, 78(2):24503, jul 2008.

[43] David J. van Woerkom, Attila Geresdi , and Leo P. Kouwenhoven. One minute parity lifetime of a NbTiN Cooper-Pair transistor. Nature Physics, 11(7):547-550, jul 2015.

[44] SuperCDMS Collaboration, R. Agnese, A. J. Anderson, T. Aramaki, M. Asai, W. Baker, D. Balakishiyeva, D. Barker, R. Basu Thakur, D. A. Bauer, J. Billard, A. Borgland, M. A. Bowles, P. L. Brink, R. Bunker, B. Cabrera, D. O. Caldwell, R. Calkins, D. G. Cerdeno, H. Chagani, Y. Chen, J. Cooley, B. Cornell, P. Cushman, M. Daal, P. C. F. Di Stefano, T. Doughty, L. Esteban, S. Fallows, E. Figueroa-Feliciano, M. Ghaith, G. L. Godfrey, S. R. Golwala, J. Hall, H. R. Harris, T. Hofer, D. Holmgren, L. Hsu, M. E. Huber, D. Jardin, A. Jastram, O. Kamaev, B. Kara, M. H. Kelsey, A. Kennedy, A. Leder, B. Loer, E. Lopez Asamar, P. Lukens, R. Mahapatra, V. Mandic, N. Mast, N. Mirabolfathi, R. A. Moffatt, J. D. Morales Mendoza, S. M. Oser, K. Page, W. A. Page, R. Partridge, M. Pepin, A. Phipps, K. Prasad, M. Pyle, H. Qiu, W. Rau, P. Redl, A. Reisetter, Y. Ricci, A. Roberts, H. E. Rogers, T. Saab, B. Sadoulet, J. Sander, K. Schneck, R. W. Schnee, S. Scorza, B. Serfass, B. Shank, D. Speller, D. Toback, R. Underwood, S. Upadhyayula, A. N. Villano, B. Welliver, J. S. Wilson, D. H. Wright, S. Yellin, J. J. Yen, B. A. Young, and J. Zhang. New Results from the Search for Low-Mass Weakly Interacting Massive Particles with the CDMS Low Ionization Threshold Experiment. Physical Review Letters, 116(7):71301, feb 2016.

[45] E. Armengaud, Q. Arnaud, C. Augier, A. Benot, A. Benot, L. Bergé, T. Bergmann, J. Billard, J. Blümer, T. de Boissière, G. Bres, A. Broniatowski, V. Brudanin, P. Camus, A. Cazes, M. Chapellier, F. Charlieux, L. Dumoulin, K. Eitel, D. Filosofov, N. Foerster, N. Fourches, G. Garde, J. Gascon, G. Gerbier, A. Giuliani, M. Grollier, M. Gros, L. Hehn, S. Hervé, G. Heuermann, V. Humbert, M. De Jésus, Y. Jin, S. Jokisch, A. Juillard, C. Kéfélian, M. Kleifges, V. Kozlov, H. Kraus, V. A. Kudryavtsev, H. Le-Sueur, J. Lin, M. Mancuso, S. Marnieros, A. Menshikov, X.-F. Navick, C. Nones, E. Olivieri, P. Pari, B. Paul, M.-C. Piro, D.V. Poda, E. Queguiner, M. Robinson, H. Rodenas, S. Rozov, V. Sanglard, B. Schmidt, S. Scorza, B. Siebenborn, D. Tcherniakhovski, L. Vagneron, M. Weber, E. Yakushev , and X. Zhang. Constraints on low-mass WIMPs from the EDELWEISS-III dark matter search. Journal of Cosmology and Astroparticle Physics, 2016(05):19, 2016.

[46] The litterature usually quote the electron equivalent sensitivity (or the energy threshold for a given bandwidth), which is about a factor 3 smaller (better) than the nuclear recoil one in $\mathrm{Ge}$, because of the larger ionization 
yield of electromagnetic interactions compared to weak interactions.

[47] A. Phipps, A. Juillard, B. Sadoulet, B. Serfass , and Y. Jin. A HEMT-Based Cryogenic Charge Amplifier with sub-100 eVee Ionization Resolution for Massive Semiconductor Dark Matter Detectors. ArXiv:1611.09712 [astroph, physics:physics], nov 2016. 